\title{
SELECTION ON SEX-RATIO AND THE EVOLUTION OF SEX-DETERMINATION
}

\author{
ILAN ESHEL \\ Department of Statistics, Tel-Aviv University, Israel
}

Received 20.vi.73

\section{SUMMARY}

Deterministic selection forces operating at a modifier level within a population lead to the establishment of systems of sex-determination which, under a variety of natural conditions, induce sex-ratios close to $1: 1$.

\section{Sex-ratio and sex-determination-the Fisherian Pringiple and its LIMITATIONS}

THE problem of how a certain sex-ratio is maintained in a given population has long attracted the attention of population biologists. Firstly, this problem has been solved in a parallel way by organisms of various groups. Secondly, in many cases the empirical data concerned are relatively easy to handle. Finally, the problem connects features at population level with cytological features of the individual-problems concerning sex-ratio are closely related to problems of sex-determination. In this context, sex-determination is used in its most general connotation as any division of a recombinant population into two self-incompatible groups. For example, when sex is determined exclusively by the $X Y$ or $X O$ system without any meiotic drive or gametic selection, a sex-ratio of $1: 1$ is manifestly established within one generation. However, this may not be the case for other systems of sex-determination (e.g. see White, 1954; Crew, 1954; Bacci, 1965). Moreover, significant deviations from a sex-ratio of $1: 1$ are theoretically possible and have been empirically recorded even in populations in which sex is determined by the $X Y$ system (e.g. Parkes, 1925; Sturtevant and Dobzhansky, 1936; Dobzhansky, 1937; Humphry, 1945; Wallace, 1948; Dunn, 1956, 1964; Morton, Chung and Mi, 1967). A classic question, dating back to Darwin (1859), see also Fisher (1958), is thus: why are sex-ratios close to $1: 1$ so common in nature? The question is of special interest since, in many natural situations, a surplus of females seem to be advantageous for the general fertility of the population. Moreover, a system determining a fixed sex ratio, especially $1: 1$, may be disadvantageous for the " economy" of the population when compared with more flexible systems, as the haploiddiploid one (see White, 1954, p. 279).

Technical explanations, based on any specific structure of a given system of sex-determination, common in nature though this system may be, are unsatisfactory on a theoretical level, since they do not explain why the system in question is so common. On the same basis, it is hard to accept an explanation based on a manifestation of the theoretical phenomenon that " most" imaginable systems, or at least the simplest ones, lead to the commonly observed sex ratio (Scudo, 1964). Actually, natural selection for statistically "uncommon" features is believed by most biologists to be the main source of evolutionary progress in nature. 
A more general argument, attempting to be independent of the system, is given by Fisher (1958, p. 158) and his followers (see Shaw, 1958; Bodmer and Edwards, 1960; Kolman, 1960; Edwards, 1960). To summarise this argument, let us consider three successive generations of a dioecious population. Suppose that the parents in the first generation produce $n_{1}$ male and $n_{2}$ female-offspring to form the parents in the second generation. These, in turn, give birth to $\mathcal{N}$ offspring of the third generation, either males or females. Since, independently of the mating system, any offspring in the third generation has exactly one male and one female parent, it is clear that any male born in the second generation will have an average of $\mathcal{N} / n_{1}$ offspring in the third generation; any female in the second generation will have an average of $\mathcal{N} / n_{2}$ offspring. (Note that these averages include potential parents which fail to reach maturity). Now, to complete the argument, compare individual parents of the same sex in the first generation, which have the same total number of direct offspring, say $m$, but with different numbers of male offspring. If $x$ is the number of male offspring and $m-x$ the number of female offspring born to such a parent, then this parent's expected number of thirdgeneration descendants is:

$$
x \frac{N}{n_{1}}+(m-x) \frac{N}{n_{2}}=\frac{m N}{n_{2}}+\left(\frac{1}{n_{1}}-\frac{1}{n_{2}}\right) N x .
$$

Obviously, this is either an increasing or a decreasing function of $x$, depending on whether the proportion of male offspring in this population is less than or more than one-half respectively, i.e. whether $n_{1}<n_{2}$ or $n_{2}<n_{1}$. Therefore, for genotypes of the same fitness (i.e. having the same expected number of viable direct offspring), the expected number of descendants to be born in the third generation is increasing with any intrinsic tendency for either sex to produce more offspring of the sex which, at the moment, is rare. With the tacit assumption that male and female parents transfer the same amount of genetic information to their progeny, this basic argument demonstrates a quite universal mechanism of selection operating toward a sex-ratio of $1: 1$. However, a more detailed consideration of the genetic structure of inheritance in a diploid population raises at least two crucial objections to the Fisherian argument.

The first objection raised by Haldane and Jayakar (1964) and later further developed by Hamilton (1967) (see also Wallace, 1948), stems from the possible inequality in the amount of relevant genetical material, transferred to the next generation through parents of different sexes. Here, by relevant we mean any genetical information that may affect the sex ratio. Actually, this is the situation whenever some relevant genetical information is located in a sex-linked locus. An important example, described by Hamilton (1967), occurs in an $X Y$ system when male gametes carrying a mutated $\Upsilon^{\prime}$ chromosome become more successful than male gametes carrying the $Y$ chromosome in fertilising the available femalegametes. In this situation it is readily shown that without continuing availability of sex-ratio modifiers (see the discussion) the " aggressive" $r^{\prime}$ chromosome will be established in the population, resulting in an increased frequency of males. The same phenomenon occurs when an aggressive $X^{r}$ chromosome is introduced into the population, except that in this case, its establishment in the population is slower. The extensively studied example 
is the female producing $X_{r}$ chromosome in Drosophila pseudoobscura (Sturtevant and Dobzhansky, 1936; Wallace, 1948).

In both cases, namely of a male and of a female producing $X$ chromosome, the danger that the population will become unisexual and thus become extinct, has been pointed out by many authors (e.g. Wallace, 1948; Haldane and Jayakar, 1964; Hamilton, 1967). Hamilton first suggested that the inactivation of the $r$ chromosome is a common cytological feature which prevents this possibility. A more complicated mechanism based on kinselection has been suggested by Hamilton as preventing the establishment of the absolutely aggressive $X$ chromosome. Note, however, that with the establishment of a moderately aggressive $X$ chromosome, any frequency of males between 0 and $\frac{1}{2}$ may be maintained, sometimes for the apparent advantage of the entire population, at least in terms of fertility. The rarity, though not absence, of this phenomenon is thus hard to explain on the basis of either kin-selection or group selection.

A model developed by Edwards (1961) explains, on a basis of differences in viabilities, the existence of a polymorphism between a normal $X$ and a female producing $X_{r}$ chromosome in Drosophila pseudoobscura. The model indeed predicts significant deviations from the Fisherian sex ratio. However, unlike the model of Hamilton, Edwards' model does not suggest any general mechanism preventing an ultimate fixation of a female (or male) reproducing chromosome, when such a mutated chromosome has no harmful effect on the viability of at least some of its carriers.

Another objection to the generality of the simple Fisherian argument, as described above, stems from its neglecting possible effects of recombinations within the system of sex-determination. Thus, in some systems wherein sex is determined by two or more loci with a positive rate of recombination between them (or, equivalently, by addition of sex-influencing loci to the $X Y$ system), the existence of a stable equilibrium with a sex ratio different from a 1 : 1 was suggested by Scudo (1964) and verified by Karlin and Gasko (personal communication). Furthermore, Karlin and Gasko have demonstrated a large variety of two-locus systems in which sex-ratios different from $1: 1$ are to be stably maintained.

In this work we are mainly interested in the question of why, despite the theoretical limitations of the Fisherian principle as mentioned above, such a principle seems to work in a rather wide variety of situations. In other words, we are interested in the question of why the genetical prerequisites for the Fisherian principle are valid in most natural systems of sex-determination. Employing essentially Fisherian methods of analysis on a modifier level, we thus attempt to show that forces within the population tend to operate toward the establishment of such systems which eventually determine a sexratio of $1: 1$.

More specifically, it is likely that the various systems of sex-determination, which are sometimes different from one species to another, are themselves determined by genetical factors which we will refer to as modifiers. If so, these factors may be, in turn, subject to selection forces within the population (see, for comparison Karlin and McGregor, 1972; Eshel, 1972). Moreover, while systems of sex-determination can be quite complicated a-priori, it is unlikely that very many of them compete within the same population. Actually, one may conceive, at the most, a single mutation in a given modifying locus, introducing one or (in a diploid population) two modified 
systems. In this case it is shown that selection within the population always favours modifying alleles which determine sex ratios which are the closest possible to $1: 1$, independent of the optimal sex ratio for the existence of the population (for a different opinion see Verner, 1965).

2. Fisherian selection over mutable systems, a biological set-up of THE MODEL AND BASIC RESULTS

In this section we suggest a model for modifications in a diploid sexdetermination system caused, one at a time, by a single mutation $A \rightarrow a$ in a single modifying locus which is not sex linked. Each of the modifying types $A A, A a$, or $a a$ determines a different system of sex-determination, possibly with a different sex-ratio intrinsically determined by the modified system. The frequency $M$ of males in the entire population is then a weighted average of the frequencies of males determined by the different systems.

More specifically, let $\alpha, \beta$ and $\gamma$ be the frequencies of males within the determined systems $A A, A a$ and $a a$ respectively. Let $p_{11}, p_{21}, p_{31}$ be the frequencies of the types $A A, A a$ and $a a$ among males, $p_{12}, p_{22}, p_{32}$ their frequencies among females. Set $p_{1}=p_{11}+\frac{1}{2} p_{21}, p_{2}=p_{12}+\frac{1}{2} p_{22} ; q_{i}=1-p_{i}$, $i=1,2$. $\quad p_{1}$ and $p_{2}$ are the frequencies of the gene $A$ among males and females respectively. Finally, assume that all males and females mate randomly, regardless of what genetical factors affect their sexes. If fitness-selection does not operate on the modifying loci we get, after one generation the frequencies in table 1 .

TABLE 1

Frequencies after one generation

Genotype

Frequency among newborn offspring

Frequency among newborn males

Frequency among newborn females
$A A$

$p_{1} p_{2}$

$$
\frac{\alpha}{M} p_{1} p_{2}
$$

$\frac{1-\alpha}{1-M} p_{1} p_{2}$
$A a$

$$
p_{1} q_{2}+p_{2} q_{1}
$$$$
\frac{\beta}{M}\left(p_{1} q_{2}+p_{2} q_{1}\right)
$$$$
\frac{1-\beta}{1-M}\left(p_{1} q_{2}+p_{2} q_{1}\right)
$$

$a a$

$q_{1} q_{2}$

$$
\frac{\gamma}{M} q_{1} q_{2}
$$

$\frac{1-\gamma}{1-M} q_{1} q_{2}$

where

$$
\begin{aligned}
M=M\left(p_{1}, p_{2}\right)=\alpha p_{1} p_{2}+\beta\left(p_{1} q_{2}+p_{2} q_{1}\right)+\gamma q_{1} q_{2} \\
=(\alpha-2 \beta+\gamma) p_{1} p_{2}+(\beta-\gamma)\left(p_{1}+p_{2}\right)+\gamma
\end{aligned}
$$

is the frequency of males in the population. If $2 \beta \neq \alpha+\gamma,(2.1)$ determines a symmetric hyperboloid over the $\left(p_{1}, p_{2}\right)$ plane. In the special additive situation $2 \beta=\alpha+\gamma$, this degenerates to a plane in the space.

From the second generation on, the population is uniquely determined by the gene-frequencies $p_{1}$ and $p_{2}$ of the $A$-allele in the previous generation. It follows immediately from table 1 , that the transformation from the gene frequencies $\left(p_{1}, p_{2}\right)$ in one generation to the gene-frequencies $\left(p_{1}^{\prime}, p_{2}^{\prime}\right)$ in the next generation is given by

$p_{1}^{\prime}=\frac{1}{M}\left[(\alpha-\beta) p_{1} p_{2}+\frac{\beta}{2}\left(p_{1}+p_{2}\right)\right]$ 


$$
p_{2}^{\prime}=\frac{1}{1-M}\left[(\beta-\alpha) p_{1} p_{2}+\frac{1-\beta}{2}\left(p_{1}+p_{2}\right)\right]=\frac{1}{1-M}\left[\frac{p_{1}+p_{2}}{2}-M p_{1}^{\prime}\right]
$$

For a quite similar model, attempting to predict the portions of "expenditure" wasted by hermaphrodites in producing male and female gametes, the reader is referred to Scudo (1964). Having assumed similar quantitative relations, the methods used here may be applicable to the model of Scudo.

For the transformation $(2.2 a-b)$ it will be shown that any stable fixed point $\left(\hat{p}_{1}, \hat{p}_{2}\right)$ either determines a male frequency of one-half or else it is symmetric $\left(\hat{p}_{1}=\hat{p}_{2}\right)$ and minimises $\left|M(p, p)-\frac{1}{2}\right|$, the deviation of the male frequencies in symmetric points from a half, at least in a local sense. A stable fixed point $\left(\hat{p}_{1}, \hat{p}_{2}\right)$ determining a sex ratio of $1: 1$ need not be symmetric, and if it is polymorphic, then in general it is not symmetric, i.e. except for a special, degenerate situation, if a stable mixture of systems determines a sex-ratio of $1: 1$, then it corresponds to different ratios of the allele $A$ in males and in females. More important, a monomorphic equilibrium $(0,0)$ or $(1,1)$ is stable if and only if it locally minimises the deviation of male frequency from $\frac{1}{2}$ (not only over symmetric points). As a special case this means that any monomorphic system determining a sex ratio of $1: 1$ is stable with respect to any possible perturbation introduced by a modifier which is not sex-linked. This, in conjunction with the fact that a sex-ratio of exactly $1: 1$ is determined by the simplest systems (Scudo, 1964), may account for the predominance of these systems in nature. We will further see that non-sex-linked modifiers are also likely to stabilise such systems against perturbations introduced in a sex-linked locus, and thus sex ratios close to $1: 1$ (but not exactly $1: 1$ ) are expected to be common in a rather wide variety of situations (see the discussion).

\section{A mathematical analysis of the model}

From (2.2) it follows that $(1-M) p_{2}^{\prime}+M p_{1}^{\prime}=\frac{p_{1}+p_{2}}{2}$. At equilibrium:

$$
\left(M-\frac{1}{2}\right) p_{1}=\left(M-\frac{1}{2}\right) p_{2} \text {. }
$$

We thus readily get:

Corollary 1. There can possibly be two kinds of equilibrium points:

(i) Symmetric equilibria with $p_{1}=p_{2}=p^{*}$ say.

(ii) Equilibrium points $\left(\hat{p}_{1}, \hat{p}_{2}\right)$ with $M\left(\hat{p}_{1}, \hat{p}_{2}\right)=\frac{1}{2}$.

We start by analysing the symmetric case. From (2.1) and (2.2a) with the postulate $p_{1}=p_{2}=p_{1}^{\prime}=p_{2}^{\prime}=p$ we obtain:

$$
p=\frac{(\alpha-\beta) p^{2}+\beta p}{(\alpha-2 \beta+\gamma) p^{2}+2(\beta-\gamma) p+\gamma}
$$

the denominator being positive for all $0 \leqq p \leqq 1$.

The two solutions $p=0$ and $p=1$ determine the trivial equilibria, corresponding to fixation of the $a$ and the $A$ gene respectively. If $2 \beta \neq \alpha+\gamma$, 
the equation (3.2) has a third solution $p^{*}$, not necessarily inside the unit square

$$
p^{*}=\frac{(\gamma-\beta)}{(\alpha-\beta)-(\gamma-\beta)} \text {. }
$$

Note that with the assumption of non-additivity $2 \beta \neq \alpha+\gamma,\left(p^{*}, p^{*}\right)$ is the centre of the hyperboloid $z=M(x, y)$. In this case $M$ may be written as

where

$$
M=(\alpha-2 \beta+\gamma)\left(p_{1}-p^{*}\right)\left(p_{2}-p^{*}\right)+M^{*}
$$

$$
M^{*}=M\left(p^{*}, p^{*}\right)=\frac{\alpha \gamma-\beta^{2}}{\alpha-2 \beta+\gamma} .
$$

Finally $0<p^{*}<1$ (and thus $\left(p^{*}, p^{*}\right)$ ) is a symmetric polymorphism of modifiers if either $\beta>\alpha, \gamma$ or $\beta<\alpha, \gamma$.

If $\beta>\alpha, \gamma(\beta<\alpha, \gamma)$ then both $M(0,0)=\gamma$ and $M(1,1)=\alpha$ are local minima (maxima) of $M\left(p_{1}, p_{2}\right)$ over the unit square (not only over symmetric points) and $M\left(p^{*}, p^{*}\right)=M^{*}$ is a global maximum (minimum) of $M(x, x)$ on the main diagonal. The former statement follows from the fact that

$$
\begin{aligned}
& \frac{\partial}{\partial p_{1}} M(0,0)=\frac{\partial}{\partial p_{2}} M(0,0)=\beta+\gamma \\
& \frac{\partial}{\partial p_{1}} M(1,1)=\frac{\partial}{\partial p_{2}} M(1,1)=\alpha-\beta .
\end{aligned}
$$

To check the stability of the corner $(0,0)$ we examine the corresponding matrix of linear (local) analyses corresponding to (2.2). If $0<\gamma<1$ we readily obtain

$$
A(0,0)=\left\|\begin{array}{ll}
\frac{\beta}{2 \gamma} & \frac{\beta}{2 \gamma} \\
\frac{1}{2} \frac{1-\beta}{1-\gamma} & \frac{1}{2} \frac{1-\beta}{1-\gamma}
\end{array}\right\|
$$

The two eigenvalues of this matrix are

$$
\begin{gathered}
\lambda_{1}=0 \\
\lambda_{2}=\frac{\beta+\gamma-2 \beta \gamma}{2 \gamma(1-\gamma)}>0 .
\end{gathered}
$$

It is easy to see that $\lambda_{2}<1$ iff $\beta<\gamma<\frac{1}{2}$ or $\beta>\gamma>\frac{1}{2}$, and $\lambda_{2}=1$ iff an equality replaces one or more of the above inequalities. Finally, if $\gamma=0$ (but $\alpha>0$ or $\beta>0$ ) or $\gamma=1$ (but $\alpha<1$ or $\beta<1$ ), instability of the corner $(0,0)$ is immediate. Hence we get:

Corollary 2a. The equilibrium $(0,0)$ is stable if

and only if

$$
\left|\gamma-\frac{1}{2}\right|<\left|\beta-\frac{1}{2}\right|
$$

$$
\left|\gamma-\frac{1}{2}\right| \leqq\left|\beta-\frac{1}{2}\right| \text {. }
$$


By symmetry we also get:

Corollary 2b. The equilibrium $(1,1)$ is stable if $\left|\alpha-\frac{1}{2}\right|<\left|\beta-\frac{1}{2}\right|$ and only if $\left|\alpha-\frac{1}{2}\right| \leqq\left|\beta-\frac{1}{2}\right|$.

In biological terms these two corollaries may be summarised in:

Corollary 2. A monomorphic system of sex-determination is locally stable if and only if a small genetical perturbation in the modifying locus, introducing a new system into the population, increases the deviation of the frequency of males in the population from a half.

To check the stability of the third possible symmetric equilibrium $\left(p^{*}, p^{*}\right)$ we write the matrix of linear (local) analysis at this point

$$
A\left(p^{*}, p^{*}\right)=\left\|\begin{array}{ll}
1-\frac{\beta}{2 M^{*}} & 1-\frac{\beta}{2 M^{*}} \\
1-\frac{1}{2} \frac{1-\beta}{1-M^{*}} & 1-\frac{1}{2} \frac{1-\beta}{1-M^{*}}
\end{array}\right\|
$$

where as we recall $M^{*}=M\left(p^{*}, p^{*}\right)=\left(\alpha \gamma-\beta^{2}\right) /(\alpha-2 \beta+\gamma)$ is the extreme value of $M$ on the main diagonal. The eigenvalues of this matrix are

$$
\begin{aligned}
& \lambda_{1}=0 \\
& \lambda_{2}=2-\frac{\beta+M^{*}-2 \beta M^{*}}{2 M^{*}\left(1-M^{*}\right)}>0 .
\end{aligned}
$$

$\lambda_{2}<1$ iff $\beta\left(1-2 M^{*}\right)>M^{*}\left(1-2 M^{*}\right)$ or, equivalently iff either

$$
M^{*}>\max \left(\frac{1}{2}, \beta\right) \quad \text { or } M^{*}<\min \left(\frac{1}{2}, \beta\right) \text {. }
$$

Note that $M^{*}$, as an extreme value of $M(x, x)$, cannot lie between the values $\alpha=M(1,1)$ and $\gamma=M(0,0)$, and that $M^{*}$ is a weighted average of the values $\alpha, \beta$ and $\gamma$. Therefore, $M^{*}>\beta\left(M^{*}<\beta\right)$ implies $\alpha, \gamma \geqq M^{*}\left(\alpha, \gamma \leqq M^{*}\right)$ with a sharp inequality if $0<p^{*}<1$. Thus it follows that $\lambda_{2}<1$ iff either

or

$$
\alpha, \gamma>M^{*}>\frac{1}{2}
$$

$$
\alpha, \gamma<M^{*}<\frac{1}{2} \text {. }
$$

In the first case $M^{*}=\min _{0 \leqq p \leqq 1} M(p, p)$, in the second case

$$
M^{*}=\max _{0 \leqq p \leqq 1} M(p, p) \text {. }
$$

$\lambda_{2} \leqq 1$ if either (3.1la) or (3.1lb) holds as a weak inequality.

The following corollary is immediately implied.

Corollary 3. A symmetric polymorphism is stable if and only if it minimises the deviation of the male frequency from one-half over all possible symmetric populations. Here, by symmetric we mean a population with the same allelic frequencies among males as among females.

Recalling that any fixed point of the process is either symmetric or corresponds to a male frequency of one-half (Corollary l), the last two corollaries lead to: 
Corollary 4. A stable equilibrium with a male frequency different from one-half can be maintained only if a male frequency of one-half is not achieved for any symmetric population $(p, p)(0 \leqq p \leqq 1)$.

With the assumption of global convergence, Corollary 4 indicates that if a male frequency of one-half is achieved for some symmetric point $(p, p)$, then there should be a stable equilibrium $\left(\hat{p}_{1}, \hat{p}_{2}\right)$, not necessarily symmetric, with $M\left(\hat{p}_{1}, \hat{p}_{2}\right)=\frac{1}{2}$. Moreover, starting from any point which is not on the corners $(0,0)$ or $(1,1)$ the process yields a limiting male frequency of onehalf.

To characterise the equilibrium points $\left(\hat{p}_{1}, \hat{p}_{2}\right)$ with $M\left(\hat{p}_{1}, \hat{p}_{2}\right)=\frac{1}{2}$, let us treat separately the additive case $2 \beta=\alpha+\gamma$ and the non-additive one $2 \beta \neq \alpha+\gamma$. In the non-additive case, we employ (3.4) to express the hyperbola $M=\frac{1}{2}$ in the form

$$
\left(p_{1}-p^{*}\right)\left(p_{2}-p^{*}\right)=\frac{\frac{1}{2}-M^{*}}{\alpha-2 \beta+\gamma}
$$

where $p^{*}$ and $M^{*}$ are defined in (3.3) and (3.5) respectively. The curve $M=\frac{1}{2}$ intersects the main diagonal $p_{1}=p_{2}$ exactly once within the unitsquare iff $\alpha-\frac{1}{2}$ is different in sign from $\gamma-\frac{1}{2}$. It intersects the main diagonal twice within the unit square iff either $\alpha, \gamma<\frac{1}{2}<M^{*}$ or $\alpha, \gamma>\frac{1}{2}>M^{*}$.

Under the condition $M=\frac{1}{2}$, the equation $p_{1}^{\prime}=p_{1}$ (with $p_{1}^{\prime}$ determined in $(2.2 \mathrm{a}))$, may be written

$$
p_{1}=2(\alpha-\beta) p_{1} p_{2}+\beta\left(p_{1}+p_{2}\right) .
$$

In the dominant situation where $\alpha=\beta$, this is the line

$$
(1-\beta) p_{1}=\beta p_{2} \text {. }
$$

If $\alpha \neq \beta$, (3.13) determines a negative hyperbola

$$
\left(p_{1}+\frac{\beta}{2(\alpha-\beta)}\right)\left(p_{2}-\frac{1-\beta}{2(\alpha-\beta)}\right)=-\frac{\beta(1-\beta)}{4(\alpha-\beta)^{2}} \text {. }
$$

The centre of this hyperbola is either in the 2 nd or in the 4th quadrant of the plane, depending on whether $\alpha>\beta$ or $\alpha<\beta$ respectively. The equilibrium points $\left(\hat{p}_{1}, \hat{p}_{2}\right)$ with $M\left(\hat{p}_{1}, \hat{p}_{2}\right)=\frac{1}{2}$ are exactly all intersections of the curves (3.12) and (3.13).

It may be shown that the two curves never intersect within the unit square if $\frac{\frac{1}{2}-M^{*}}{\alpha-4 \beta+\gamma}<0$. On the other hand, if $\frac{\frac{1}{2}-M^{*}}{\alpha-2 \beta+\gamma} \geqq 0$, then the two curves always intersect within the unit square. In this case there are at the most two points of intersection. The proof is technical and will be omitted. It is readily shown that except for the special case

$$
2 \beta(1-\beta)=\alpha(1-\gamma)+\gamma(1-\alpha)
$$

(and, thus, $M^{*}=\frac{1}{2}$ and $\hat{p}_{1}=\hat{p}_{2}=p^{*}$ ) the intersections are at asymmetric points $\hat{p}_{1} \neq \hat{p}_{2}$.

It remains to study the special situation of complete additivity $2 \beta=\alpha+\gamma$ $(\alpha \neq \gamma)$. In this case we know that no symmetric polymorphism $\left(p^{*}, p^{*}\right)$ exists and the equation $M(x, y)=\frac{1}{2}$ corresponds to the real line

$$
x+y=\frac{1-2 \gamma}{\alpha-\gamma} .
$$


Without loss of generality assume $\alpha>\gamma$. The line (3.14) then intersects the unit square iff

$$
\gamma<\frac{1}{2}<\alpha
$$

Note that with the assumption $2 \beta=\alpha+\gamma, \alpha>\gamma,(3.15)$ is also a necessary and sufficient condition for instability of both corners $(0,0)$ and $(1,1)$. Non-symmetric equilibria, if they exist, are exactly the intersections of $M\left(p_{1}, p_{2}\right)=\frac{1}{2}$ with the hyperbola

$$
p_{1}=2(\alpha+\beta) p_{1} p_{2}+\beta\left(p_{1}+p_{1}\right)=(\alpha-\gamma) p_{1} p_{2}+\frac{\alpha+\gamma}{2}\left(p_{1}+p_{2}\right) .
$$

It is easily verified that the condition (3.15) is a necessary and sufficient condition for the existence of a solution in the square unit. The solution is then unique, given by

$$
\begin{aligned}
\hat{p}_{1} & =\frac{\frac{\sqrt{2}}{2} \sqrt{\alpha+\gamma-2 \alpha \gamma-\gamma}}{\alpha-\gamma} \\
\hat{p}_{2} & =\frac{1-\gamma-\frac{\sqrt{2}}{2} \sqrt{\alpha+\gamma-2 \alpha \gamma}}{\alpha-\gamma} .
\end{aligned}
$$

With the assumption of global convergence, the findings of this section may be summarised in the following theorem.

(i) All fixed points of the process are either symmetric or determine a sex-ratio of $1: 1$. Except for the special case $M^{*}=\frac{1}{2}$, points of the second type are not symmetric, corresponding to different gene-frequencies among males and females.

(ii) Symmetric fixed points are stable if and only if they minimise the deviation of male frequencies from one-half at least over symmetric points and in a local sense. If they are monomorphic, they also locally minimise the deviation over non-symmetric points. A symmetric stable polymorphism, if it exists, globally minimises the symmetric deviation of the sex-ratio from $1: 1$.

(iii) A stable (generally non-symmetric) fixed point determining a sexratio of $1: 1$ exists if and only if there is at least one symmetric population $(p, p)$ (generally not in equilibrium) with a sex-ratio $M(p, p)=\frac{1}{2}$.

(iv) If a monomorphic system of sex determination maintains a sex-ratio of $1: 1$, then it is stable with respect to all possible small perturbations which are not determined by sex-linked modifiers.

(v) A monomorphic system determining a sex-ratio different from 1:1 is always unstable with respect to non-sex-linked modifiers which shift the sex-ratio toward $1: 1$.

Despite the close similarity between Fisherian fitness-selection and the process described here, not that the equivalent of Fisher's fundamental law of fitness does not hold for Fisherian selection for a sex-ratio of $1: 1$, even in the case of a single locus. Although the limit points of the iterated transformation appear to minimise the deviation from a sex-ratio of $1: 1$, the transformation (2.2) may temporarily increase this deviation, a fact which makes the analysis of the global convergence complicated. 
For, suppose $\alpha<\frac{1}{2}<\gamma, 2 \beta \neq \alpha+\gamma$. In this case, the equation $M=\frac{1}{2}$ determines a curve of points, only two of which may be fixed points. For all other points on the curve (2.2) readily imply $p_{1}^{\prime}+p_{2}^{\prime}=p_{1}+p_{2}$ but $p_{1}^{\prime} p_{2}^{\prime} \neq p_{1} p_{2}$. From (2.1) we thus get:

$$
\left|M^{\prime}-\frac{1}{2}\right|=|\alpha-2 \beta+\gamma|\left|p_{1}^{\prime} p_{2}^{\prime}-p_{1} p_{2}\right|>0=\left|M-\frac{1}{2}\right|
$$

\section{Discussion}

We have seen that, under the limitation of a fixed system of sexdetermination, the Fisherian principle of selection on the third generation toward a sex-ratio of $1: 1$ does not always hold. The situation is different, however, when we take into consideration the fact that the very system of sex-determination is likely to be determined by genetical modifying factors. These, when mutated, may alter the whole system and thus change the sex-ratio determined in the population. In this case it has been shown that in any locus which is not sex-linked, a modifying mutation is established if and only if it shifts the induced sex-ratio toward $1: 1$. This, in conjunction with the simplicity of systems determining a sex-ratio of exactly $1: 1$ (Scudo, 1964) may account for the fact that such systems are prevalent in so many populations, presumably most of the time.

Actually, within the limitations of the model developed here, it appears that once a system determining a sex-ratio of $1: 1$ is established, deviation from this sex-ratio could occur only through mutation in sex-linked modifiers, if these exist (Haldane and Jayakar, 1964). On the basis of this analysis, however, one would expect such deviations to be of a temporal nature, sooner or later being corrected by non-sex-linked modifiers.

Empirical hints for the dynamics of the process guaranteeing sex-ratios close to $1: 1$ in newborn offspring is suggested by recent findings indicating significant deviations from the expected sex-ratio in the embryo stage in some populations in which sex is apparently determined by the $X Y$ system and sex-ratio is known to be close to $1: 1$. However, these findings are not yet significant. (This includes human populations, see Crew, 1954; Stern, 1960; White, 1965; Cavalli-Sforza and Bodmer, 1971.)

Note that kin-selection, as suggested by Hamilton (1967), may produce the same effect as non-sex-linked modifiers, especially in extreme situations in which a drastic deviation in sex-ratio immediately endangers the existence of the population. In most situations, however, an explanation based on kin-selection is not needed. On the contrary, a sex-ratio of approximately $1: 1$ is prevalent in many cases in which it is apparently far from being optimal either for the survival of the population or its overall fertility.

I do believe, however, that kin-selection, as well as the neighbour effect (Eshel, 1971) and, possibly, group selection (Wynne-Edwards, 1962) or clone-selection (Eshel, 1972) are more important in explaining the few examples in which sex-ratios significantly different from $1: 1$ are found. In these cases, sex-linked modifiers may cause a temporal deviation from a sexratio of $1: 1$. If this deviation is advantageous for the group, it may provide material for group selection which, in turn, could arrest any correcting effect of non-sex-linked modifiers. This may also prove a plausible ad-hoc answer to the crucial question raised by Williams (1966) in objection to over- 
estimation of the importance of group-selection in nature; that is, how an individually disadvantageous feature can possibly be established in any group so that group-selection can begin to operate.

Acknowledgments.-I wish to thank Professor Samuel Karlin, Dr John Karon and Mr Usi Motro for their remarks on the manuscript. Also, I would like to thank Professor Adi Zemah of the Department of Philosophy at the Hebrew University, for stimulating discussions.

\section{References}

BaCCI, G. 1965. Sex Determination (1st ed.). Pergamon Press, Oxford, N.Y.

BODMER, w. F., AND EDWARds, A. w. F. 1960. Natural selection and sex ratio. Ann. Hum. Genet., 24, 239-244.

CAvalli-sforza, L. L., And bodmer, w. F. 1971. The Genetics of Human Populations. Freeman \& Co., San Francisco.

CRew, F. A. 1954. Sex Determination (3rd ed.). Methuen, London.

DARWIN, c. 1859. The Origin of Species. John Murray, London.

DOBZHANSKX, тн. 1937. Further data on the variation of the Y-chromosome in Drosophila pseudoobscura. Genetics, 22, 340-346.

DunN, L. c. 1956. Analysis of a complex gene in the house mouse. Cold Spring Harbor Symp. Quant. Biol., 21, 187-195.

DUNN, L. C. 1964. Abnormalities associated with a chromosome region in the mouse. Science, 144, 260-263.

EDWARDS, A. W. F. 1960. Natural selection and sex ratio. Nature, 188, 960-961.

EDWARDs, A. W. F. 1961. The population genetics of "sex ratio" in Drosophila pseudoobscura. Heredity, 16, 291-304.

eshel, I. 1971. On the neighbor effect and the evolution of altruistic traits. Theor. Pop. Biol., 3 (3), 258-272.

eshel, I. 1972. Clone selection and the evolution of modifying features. Theor. Pop. Biol., 4, 196-203.

FISHER, R. A. 1958. The Genetical Theory of Natural Selection, 2nd ed. Dover, N.Y.

HALDANE, J. B. s., AND JAYAKAR, s. D. 1964. Equilibrium under natural selection at a sexlinked locus. 7. Genet., 59, 29-36.

HAMILTON, w. D. 1967. Extraordinary sex-ratio. Science, 156, 3744, 477-488.

HUMPHRY, R. R. 1945. Sex determination in Ambistomid salamanders: A study of the progeny of females experimentally converted into males. Amer. F. Anat., 76, 33-66.

KARLIN, s., AND MCGRegor, J. 1972. The role of modifier genes in evolution. Proc. Nat. Acad. Sci. U.S.A., 69, 2611-3614.

xolman, w. A. 1960. The mechanism of natural selection for the sex-ratio. Amer. Nat., 97, 373-377.

MORTON, N. E., CHung, c. s., AND MI, M. P. 1967. Genetics of Interracial Crosses in Hawaii. S. Karges, N.Y.

PARKES, A. S. 1925. Studies on the sex-ratio and related phenomena. III. The foetal sex-ratio in the pig. F. Agric. Sci., 15, 284-299.

scudo, F. M. 1964. Sex population genetics. La Ricerca Scientifica, 34, II-B, 93-146.

SHAw, R. F. 1958. The theoretical genetics of the sex-ratio. Genetics, 43, 149-163.

stern, c. 1960. Principles of Human Genetics (2nd ed.). W. H. Freeman \& Co., San Francisco.

Sturtevant, A. H., AND DOBzhansky, TH. 1936. Geographical distribution and cytology of sex-ratio in Drosophila pseudoobscura and related species. Genetics, 21, 473-490.

verner, J. 1965. Selection for sex-ratio. Amer. Nat., 99, 419-421.

wallace, B. 1948. Studies in sex-ratio in Drosophila pseudoobscura. I. Selection and sexratio. Evolution, 2, 189-217.

white, м. J. D. 1954. Animal Cytology and Evolution (2nd ed.). Cambridge Univ. Press, Oxford.

williams, G. c. 1966. Adaptation and Natural Selection. Princeton Univ. Press, Princeton, N.J.

WYNNE-EDWARd, v. c. 1962. Animal Dispersal in Relation to Social Behavior. Hafner, N.Y. 